\title{
A damage-reduced process revealed by photoluminescence in photoelectrochemical etching GaN
}

\author{
J. M. Hwang, J. T. Hsieh and H. L. Hwang \\ Department of Electrical Engineering, National Tsing-Hua University, \\ Hsin-Chu 300, Taiwan, R.O.C. \\ W. H. Hung \\ Synchrotron Radiation Research Center, Hsin-Chu 300, Taiwan, R.O.C.
}

\begin{abstract}
Photoelectrochemical (PEC) etching technique has been proven to be an effective method to etch GaN. Despite its success, investigations on etching-induced damage are still scare. In this work, the damage induced by PEC etching of $\mathrm{GaN}$ in $\mathrm{KOH}$ electrolyte was studied. Photoluminescence (PL) spectroscopy was used to explore the origin of etching-induced damaged layer. From the variable temperature PL measurements, the origin of etching-induced damage was attributed to be the defect complex of $\mathrm{V}_{\mathrm{Ga}}-\mathrm{O}_{\mathrm{N}}$ (gallium vacancy bonds to oxygeon on nitrogen antisite). With determination of the defect origin, the electronic transition in the etch damage-related yellow luminescence (YL) band was suggested to be deep donor-like state to shallow-acceptor transition. In addition, a post-treatment method with boiled $\mathrm{KOH}$ chemical etching was developed to remove the thin damaged layer. In this method, crystallographic etching characteristics of boiled $\mathrm{KOH}$ was observed to assist in the formation of smooth sidewall facets. As revealed by the reduction of yellow luminescence, we propose this novel technique as a near damage-free etching method.
\end{abstract}

\section{Introduction}

Since GaN is chemically stable and insoluble in all mineral acid and base solutions at room temperature [1], most processing of III nitrides are currently done by dry plasma etching. High density plasma or energetic ion assisted etching were used to get a smooth etch surface and highly anisotropic sidewalls with high etch rates [2-4]. But there are several disadvantages to use dry etching, including generation of the ion-induced damage and difficulty in obtaining smooth etched sidewalls, which are required for the lasers cavity. The optical damage relative to yellow luminescence (YL) was also extensively investigated using photoluminescence spectroscopy on the etched surfaces. [5] The etching-induced damage will have detrimental effect on electronic or photonic device performance. Therefore, a reliable fabrication process producing low etch damage for GaN-based devices is necessitated.

Photoelectrochemical (PEC) etching is an alternative method to produce smooth surfaces and vertical sidewall facets without ion bombardment induced damages.[6-8] As it is well-known that the yellow luminescence commonly existing in epitaxial grown GaN is related to the intrinsic defects or impurities. In this study, we used YL intensity variation to identify the etching-induced damages. A novel boiled $\mathrm{KOH}$ post treatment process was 
also developed to remove the etching-induced damages and achieve vertical and smooth facet sidewalls. A near damage-free etching process with vertical sidewalls and smooth etched surface can be realized by this post treatment process. This etching process could be applied to device fabrication such as laser cavity formation.

\section{Experimental}

The unintentionally-doped $\mathrm{n}$-GaN samples (conc. $<5^{*} 10^{16} \mathrm{~cm}^{-3}$ ) used in this study were obtained from CREE Research, Inc. The thickness of nitride layer is $1.4 \mu \mathrm{m}$. Standard lift-off procedure was used to form the Ti metal mask that it is served as a contact for better photocurrent conduction. The GaN samples were sequentially cleaned by methanol and acetone and then rinsed with deionized water. A sample holder with two Teflon plates was used to fix the GaN samples. The PEC etching of GaN was carried out in a Teflon electrochemical cell containing an aqueous $\mathrm{KOH}$ electrolyte. The UV source was an Oriel high-pressure $\mathrm{Hg}$-arc lamp, in which a water filter was mounted on the output window to remove infrared irradiation. The post-treatment was done with boiled $\mathrm{KOH}$ solution. For all photoluminescence (PL) measurements, a He-Cd laser $(\lambda=325 \mathrm{~nm})$ , typically operated at $1 \mathrm{~mW}$ output power and focused to a spot with a diameter of 100 $\mu \mathrm{m}$ on the sample surface, was used as the excitation source. The luminescence was collected by a $50 \mathrm{~mm} \mathrm{f} / 1.4$ lens in the direction normal to the illuminated surface, and then focused into a SPEX 500M monochromator with an electrical-cooled GaAs photomultiplier. The etching profile was measured by HITACHI S-40000 FESEM.

\section{Results and discussion}

Our experimental results indicate that the etching rate is linearly proportional to the power density and it increases with increasing the $\mathrm{KOH}$ concentration. The relation of etching rate v.s. power density and $\mathrm{KOH}$ concentration is very similar to the published results [2-4]. Photoluminescence spectra corresponding to the etching condition with various UV power densities are shown in Figure 1, in which the 470W, 600W and 700W UV power corresponds to the power densities of $195 \mathrm{~mW} / \mathrm{cm}^{2}, 240 \mathrm{~mW} / \mathrm{cm}^{2}$ and 290 $\mathrm{mW} / \mathrm{cm}^{2}$, respectively. All PL spectra are normalized to the same intensity scale of nearband-edge (NBE) peak at $364 \mathrm{~nm}$. Spectrum A in Figure 1 shows the room temperature (R.T.) PL of the as-grown samples.
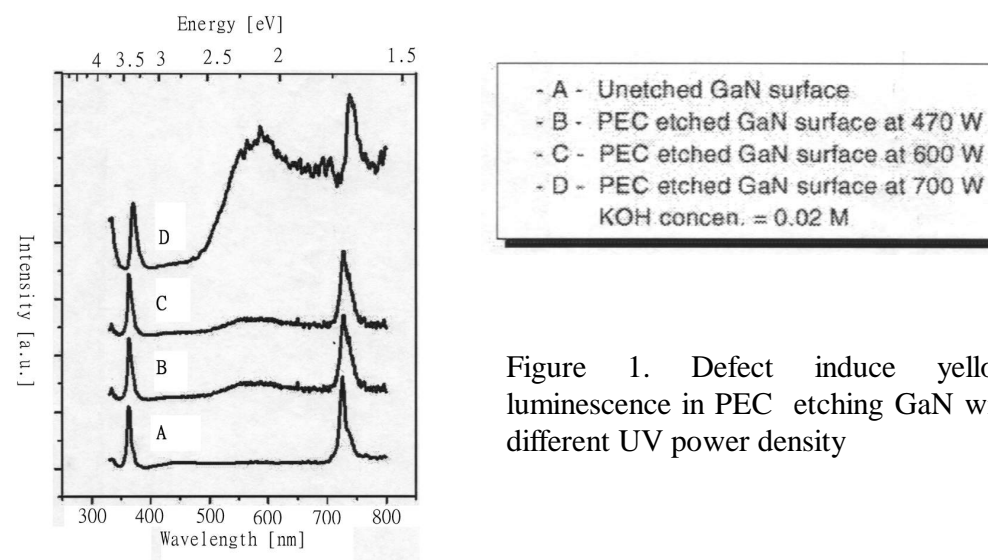

Figure 1. Defect induce yellow luminescence in PEC etching $\mathrm{GaN}$ with different UV power density 

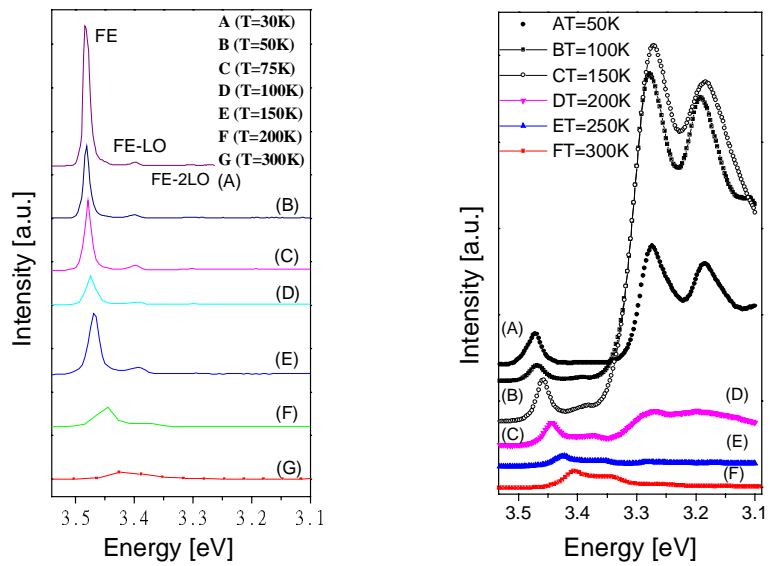

Figure 2. Temperature dependence photoluminescence of GaN (a) as- growth GaN with PL peaks as FE and phonon replica. (b)PEC etching GaN with PL peaks as FE, dislocation, DAP and phonon replica.

The peak at $364 \mathrm{~nm}(3.41 \mathrm{eV})$ is attributed to be the NBE emission and the $728 \mathrm{~nm}$ $(1.705 \mathrm{eV})$ is the second harmonic counterpart. After etching with UV power density of $195 \mathrm{~mW} / \mathrm{cm}^{2}$ in $0.02 \mathrm{M} \mathrm{KOH}$ electrolyte, a yellow luminescence band centered at $563.6 \mathrm{~nm}$ $(2.2 \mathrm{eV})$ appeared (spectrum B). In spectrum C, the yellow luminescence band remained the same as in spectrum B while increasing the power density to $240 \mathrm{~mW} / \mathrm{cm}^{2}$. With the successive increase of the power density to $290 \mathrm{~mW} / \mathrm{cm}^{2}$ for a longer etching, the very strong YL dominated and a dislocation-related peak at $370 \mathrm{~nm}(3.351 \mathrm{eV})$ emerged [9] as shown in spectrum $\mathrm{D}$. This means that the etched GaN surfaces were seriously damaged such that the YL-related recombination centers were formed to annhilate the excitonrelated luminescence due to trapping of excitons by the etching-induced defect traps. The YL relative to etching-induced damage will increase when etching $\mathrm{GaN}$ in the condition of higher power and/or longer time. To investigate origin of the etching-induced YL band emission, variable temperature PL measurements were done. Figure 2(a) shows the temperature-dependent PL of as-grown GaN samples. As were also observed by other authors, the free exciton (FE) peak position shifted to higher energy (blue shift) with decreasing temperature. The temperature coefficient of $\mathrm{FE}$ peak was determined to be $2.93 \times 10^{-4} \mathrm{eV} / \mathrm{K}\left(100^{\circ} \mathrm{K}<\mathrm{T}<300^{\circ} \mathrm{K}\right)$. Two regular LO-phonon replicas FE-LO and FE-2LO were observed in Figure 2(a) due to dominance of the polariton-phonon scattering at low temperature [10]. After PEC etching of GaN with UV power density of $322 \mathrm{~mW} / \mathrm{cm}^{2}$ and $\mathrm{KOH}$ concentration of $0.05 \mathrm{M}$ for 30 minute, the R.T. PL spectrum exhibited a new emission that is assigned to be donor-acceptor-pair (DAP) transition. The corresponding variable temperature PL spectra are shown in Figure 2(b). By successively decreasing the sample temperature to $200^{\circ} \mathrm{K}$ and below, an obvious enhancement of DAP emission was observed. Compared to the NBE transition, the peak position of DAP was less temperature-dependent [11] and was pinned at $3.229 \mathrm{eV}$. Moever, the photon from DAP transition can interact with the phonon to create the DAP-

F99W11.73 


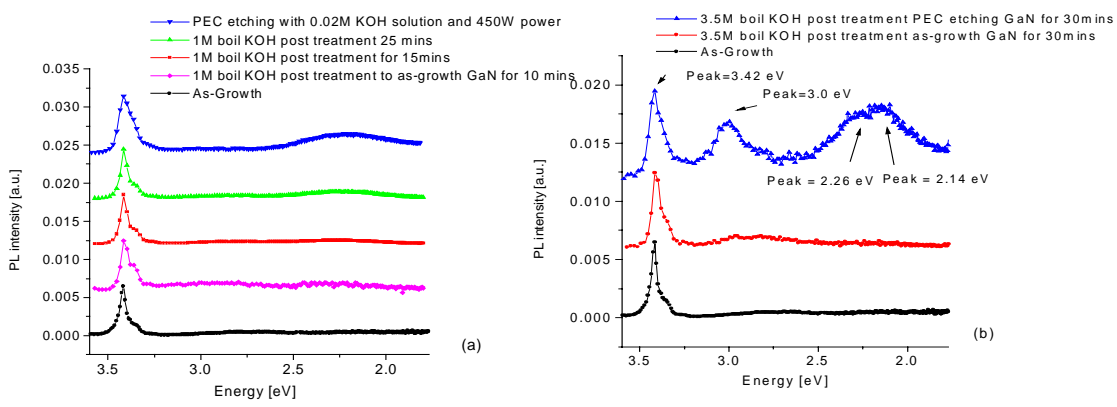

Figure 3. Damage remove by PEC etching GaN (a) low concentration of boil KOH with remove the damage layer produced YL in short time treatment. (b) high concentration on of boil $\mathrm{KOH}$ and low time treatment will create damage to $\mathrm{GaN}$ surface

LO coupling with the gain or loss of one phonon energy. At temperature below $100 \mathrm{~K}$, the LO phonon energy is about $91 \mathrm{meV}$ that is consistent with the observation of the A1 (LO) mode $\left(734.75 \mathrm{~cm}^{-1}\right)$ in our FTIR measurement. In addition, we also found that the variation of YL intensity is connected to DAP emission intensity, that is, the YL intensity increased as the DAP emission intensity increased. This seems to imply that these two radiative recombination paths share the same electronic transition levels. In the following paragraph, we will dedicate to build a defect model to explain the observed phenomena in our experiments.

In as-grown GaN film, a weak YL band exists too. It appears to have the same origin as the induced defects during PEC etching in our experiment. YL involves transitions from a deep state in the upper half of the gap either to the valence band, or to a (relatively) shallow acceptor. They are also termed as the shallow-donor to deep-acceptor transition and the deep donor-like to shallow-acceptor transition. The complex defect $\mathrm{V}_{\mathrm{Ga}^{-}}{ }^{-}$ $\mathrm{O}_{\mathrm{N}}$ create a deep donor-like state (DD) above the VBM about $2.2 \mathrm{eV}$. The excited carriers escape from the shallow donor states created by $\mathrm{V}_{\mathrm{N}}$ (Nitrogen vacancy) to $\mathrm{DD}^{-}$state in a nonradiative recombination process and then to a shallow acceptor with the YL emission $(2.2 \mathrm{eV})$ [12]. Our results support this model and the induced defect during PEC etching process were suggested to be Ga vacancy bonds to oxygen in the nitrogen site. The possible reaction mechanism is as follows. The $\mathrm{OH}^{-}$coming from $\mathrm{KOH}$ electrolyte prefers to react with $\mathrm{Ga}$ rather than $\mathrm{N}$ to form gallium oxide that subsequently dissolves into the solution. A gallium vacancy will be therefore created to bond with the neighboring antisite defects arising from the occupation of nitrogen sites by the oxygen atoms. As seen from the temperature-dependent PL spectrum, a YL transition from $\mathrm{DD}^{-}$to a shallow acceptor level was identified. At low temperature, this transition was quenched. The excited carriers follow another recombination path from a shallow donor state to a shallow acceptor state, that is, the DAP transition. The YL intensity is strongly dependent on the amount of the acceptor states. The less the acceptor states, the weaker the YL intensity, and therefore the weaker then DAP peak intensity. This coincides with our results. From the above discussion, we would suggest that the defect is the defect complex of $\mathrm{V}_{\mathrm{Ga}}-\mathrm{O}_{\mathrm{N}}$ rather just 
$\mathrm{V}_{\mathrm{Ga}}$. Further support for the energy position assignment of the observed YL stems from the AES spectra.

The surface composition of as-growth with Ga:N (1.49:1) sample. From the PL of the etching surface with less YL, the surface composition of Ga:N (1.22:1) shown. While strong YL is created by PEC etching, the surface composition of Ga decreased with GaN (1.11:1). From the surface composition analysis, we can find that the surface gallium decreases while the YL intensity increases and the surface oxygen increases while YL intensity increases. This indicates that the YL intensity will increase as the concentration of $\mathrm{V}_{\mathrm{Ga}}-\mathrm{O}_{\mathrm{N}}$ defect complex increases.

The damaged layer induced by PEC etching of GaN was stable and can not be removed by the conventional chemical etching methods such as $\mathrm{HCl}: \mathrm{H}_{2} \mathrm{O}(1: 1), \mathrm{HF}: \mathrm{H}_{2} \mathrm{O}$ $(1: 1)$ or mixed solution at room temperature or elevated temperature. This can be confirmed from the PL measurement in which the YL band still exists after various chemical treatments as listed above. A novel skill with a post-treatment process in boiled $\mathrm{KOH}$ solution was evaluated for damage removed. In Figure 3(a), the PL spectrum from as-grown GaN samples was shown. No YL band is found on the as-grown samples. After PEC etching with power density of $186 \mathrm{~mW} / \mathrm{cm}^{2}$ and $\mathrm{KOH}$ concentration of $0.02 \mathrm{M}$, the defect complex $\mathrm{V}_{\mathrm{Ga}}-\mathrm{O}_{\mathrm{N}}$ relative to $\mathrm{YL}$ was present. After $1 \mathrm{M}$ boiled $\mathrm{KOH}$ post-treatment for 10 minutes, the YL intensity was largely reduced. After 15 minutes of treatment, the PL spectrum also showed a weaker YL intensity. This means that the etching-induced damage by PEC etching of $\mathrm{GaN}$ has been removed after the hot $\mathrm{KOH}$ post-treatment. But after 25 minutes of treatment, YL band emission recovered again. The possible reason is that the boiled $\mathrm{KOH}$ start to etch GaN after removal of the damaged layer and it will produce YL related defects as the PEC etching did before. To identify the damages created by $\mathrm{KOH}$ solution in the condition of high concentration and long times, $3.5 \mathrm{M}$ $\mathrm{KOH}$ was used to etch $\mathrm{GaN}$ for 30 minutes. The PL spectra for the etched and unetched regions are shown in Figure 3(b). It can be clearly seen that blue luminescence (BL) band centered at $3.0 \mathrm{eV}$ emerges besides the YL band with peaks at $2.2 \mathrm{eV}$ and $2.14 \mathrm{eV}$. The appearance of BL band seems to reveal the creation of a new type of etching-induced defect. The origin of the BL remains as an open question. Based on above results, the boiled $\mathrm{KOH}$ post-treatment with lower concentrations and/or for shorter time is suggested to remove the damaged layer roduced by the PEC etching of GaN.

Another property of crystallographic wet etching in boiled $\mathrm{KOH}$ was useful for device processing and laser cavity formation. [13] The boiled $\mathrm{KOH}$ stops etching $\mathrm{GaN}$ at $\{10-10\}$ and (0001) faces and prefers etching dislocation sites. As shown in Figure 4(a), the stripped sidewall and residual dislocations on the etched surface of (0001) will limit the application to electronic or optical devices. To eliminate this limitation, boiled $\mathrm{KOH}$ was used to treat the PEC etched surface and the result is shown in Figure 4(b). The sidewall was smoothed and the dislocations were removed after the post-treatment by boiled $\mathrm{KOH}$. By virtue of careful control of etch time and $\mathrm{KOH}$ concentration, a very smooth and vertical facet was achieved as shown in Figure 4(c) at $\{10-10\}$ face. The striation on sidewall edge was resulted from pattern transfer during the lithography step.

\section{Conclusions}

In summary, YL-related defects induced during the PEC etching process were 

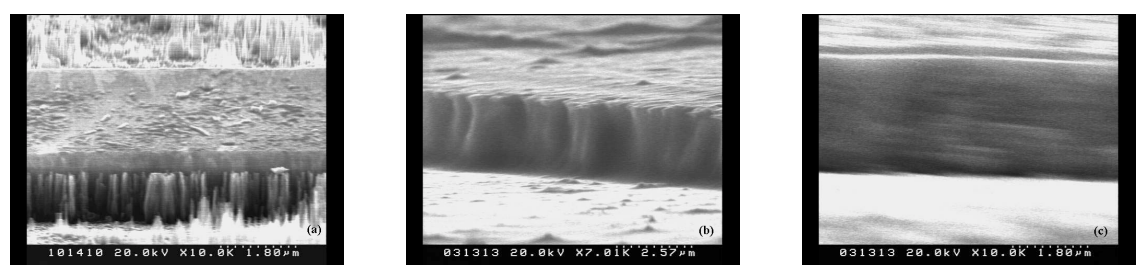

Figure 4. SEM of PEC etching GaN (a) PEC etching with sidewall roughness and dislocation residual at the etching surface (b)boil $\mathrm{KOH}$ solution post-treatment to remove residual dislocation (c) a vertical and smooth etching profile by crystallography etching with boil $\mathrm{KOH}$. solution

studied. By using PL spectroscopy, the defects were suggested to be $\mathrm{V}_{\mathrm{Ga}}-\mathrm{O}_{\mathrm{N}}$. After post-treatment in a boiled $\mathrm{KOH}$ solution with lower concentration for a short time, the thin damaged layer was removed. With help of the crystallographic wet etching in boiled $\mathrm{KOH}$ solution, smooth etch surfaces and facet sidewalls can be achieved. The residual dislocations are also taken off by boiled $\mathrm{KOH}$. This novel process provides a near damagefree etching for electronic and optical device application.

\section{Acknowledgements}

Some samples was provided by Opto-Electronics and System Laboratories, Industrial Technology Research Institute. Financial support was provided by the and National Science Council of Republic of China (Contract No. NSC 88-2215-E-007-011).

\section{References}

1. C. B. Vartuli, S. J. Pearton,C. R. Abernathy, J. D. MacKenzie, F. Ren, J.C. Zolper, R. J. Shul, SOLID-STATE ELECTRONICS vol 41, 1947-1951 (1997)

2. R. J. Shul, A. J. Howard, S. J. Pearton, C. R. Abernathy, C. B. Vartuli, P. A. Barnes and M. J. Bozack, J. Vac. Sci. Technol. B 132016 (1995)

3. A. T. Ping, A. C. Schmitz, and I. Adesida, J. of Electro. Mater. 25825 (1995)

4. Charles R. Eddy, Jr., MRS Internet J. Nitride Semicond. Res. 4S1, G10.5 (1999)

5. J. Y. Chen, C. J. Pan, and G. C. Chi, Solid-state Electronics 43649 (1999)

6. M. S. Minskey, M. White, and E. L. Hu, Appl. Phys. Lett. 68, 1531 (1996)

7. C. Youtsey, I. Adesida, and G. Bulman, Appl. Phys. Lett. 71, 2151 (1997)

8. C. Youtsey, I. Adesida, L. T. Romano, and G. Bulman, Appl. Phys. Lett. 72, 560 (1998)

9. J. T. Hsieh, J. M. Hwang, W. H. Hung and H. L. Hwang, (unpublished)

10. D. Kovalev, B. Averboukh,D. Volm, and B. J. Meyer, Phys. Rev. B 54, 2518. (1996).

11. Takashi Matsumoto and Masaharu Aoki, Japan. J. Appl. Phys. 131804 (1974)

12. E. R. Glaser, T. A. Kennedy, K. Doverspike, L. B. Rowland, D. K. Gaskill., D. T. Olson, J. N. Kuznia, and D. K. Wickenden, Phys. Rev. B 51,13326 (1995)

13. D. A. Stocker, E. F. Schubert and J. M. Redwing, Appl. Phys. Lett. 73, 2654 (1998) 